\title{
Siete nuevos registros de macroalgas para el archipiélago Diego Ramírez (56 31'S): el valor del nuevo parque marino como sumidero de carbono $y$ conservación de la biodiversidad subantártica
}

Seven new macroalgas records for the Archipelago Diego Ramirez (56 $\left.31^{\prime} S\right)$ : the value of the new marine park as a sum of carbon and conservation of subantartic biodiversity

Johanna Marambio ${ }^{1,2}$, Sebastián Rosenfeld ${ }^{1,2}$, Juan Pablo Rodríguez ${ }^{1,2}$, Fabio Méndez ${ }^{1,2}$, Tamara Contador ${ }^{3,4}$, Roy Mackenzie ${ }^{2}$, Bernard Goffinet ${ }^{5}$, Ricardo Rozzi ${ }^{2,4,6}$ \& Andrés Mansilla ${ }^{1,2}$

\section{Resumen}

Las ecorregiones marinas de canales y fiordos del sur de Chile subantártica de Magallanes representan el límite extremo de latitud sur para la distribución de numerosos grupos de organismos. En el caso del Hemisferio Sur, el punto de distribución más austral de representantes de las formaciones de "kelps" se encuentra en el archipiélago Diego Ramírez, donde se registran las especies Lessonia flavicans, $L$. searlesiana y Macrocystis pyrifera. El archipiélago Diego Ramírez se encuentra ubicado en el Paso Drake a $112 \mathrm{~km}$ al SO del Cabo de Hornos. Con respecto a la información disponible para macroalgas se ha informado un total de 79 taxa, de los cuales muchos presentan afinidad subantártica-antártica. Pese al interés biogeográfico de las macroalgas del archipiélago Diego Ramírez, no ha habido nuevos catastros que permitan evaluar si este archipiélago podría representar el extremo norte de distribución de especies antárticas y/o el extremo sur de especies magallánicas. El objetivo de este trabajo es actualizar el catastro de macroalgas del archipiélago con el fin de detectar nuevos taxa que pudieran estar presentes en estas islas, así como contribuir al análisis de las relaciones biogeográficas de su ficoflora. De la campaña realizada en la isla Gonzalo se identificó un total de 19 taxa de macroalgas. En términos de riqueza por división, Chlorophyta estuvo representada por 3 especies (16\%), Ochrophyta por 7 taxa (37\%) y Rhodophyta por 9 taxa, representando el $47 \%$ de la flora identificada. Con estos nuevos registros y los de trabajos previos, nuestro estudio genera un catastro actualizado de macroalgas para el archipiélago que concluye en la presencia de 86 taxa. En general, se pudo observar que las especies registradas durante este estudio y los trabajos previos analizados presentan afinidad subantártica.

\section{Palabras clave:}

macroalgas, subantártico, Diego Ramírez, afinidad geográfica.

\section{Abstract}

The marine ecoregions of Canals and Fjords of Southern Chile and Magellan Sub-Antarctic represent the extreme southern latitude limit for

\footnotetext{
Laboratorio de Ecosistemas Marinos Antárcticos y Subantárcticos (LEMAS), Universidad de Magallanes, Casilla 113-D, Punta Arenas, Chile; doctorante en la University of Bremen. https://orcid.org/0000-0003-1958-0351 \marambio@uni-bremen.de

2 Instituto de Ecología y Biodiversidad (IEB), Casilla 653, Santiago, Chile.

3 Laboratorio Wankara de Ecosistemas Dulceacuícolas Subantárticos y Antárticos, Universidad de Magallanes \& Instituto de Ecología y Biodiversidad (IEB), Puerto Williams, Chile.
}

4 Programa de Conservación Biocultural Subantártica, Universidad de Magallanes, Teniente Muñoz 396, Puerto Williams, Chile.

5 Department of Ecology and Evolutionary Biology, University of Connecticut, 75 North Eagleville road, Conneticut, EE.UU.

6 Department of Philosophy and Religion, University of North Texas, Dallas, EE.UU. 
the distribution of numerous organism group. In southern Hemisphere, the most austral distribution site of kelps formation is placed at Diego Ramírez Archipelago, where Lessonia flavicans, $L$. searlesiana and Macrocystis pyrifera have been registered. Diego Ramírez Archipelago is located in the Drake Passage, $112 \mathrm{~km}$ southwest to Cape Horn. Regarding the available information for macroalgae, a total of 79 taxa has been reported, many of which show a sub-Antarctic and Antarctic affinity. Despite the biogeographic interest of the macroalgae from Diego Ramírez Archipelago, there have not been new cadasters to evaluate whether this archipelago could represent the northern boundary of the Antarctic species distribution and/ or the southern boundary of Magellan species. The goal of this study is to update the cadaster of macroalgae from Diego Ramírez Archipelago, in order to find new taxa and contribute to the analysis of the biogeographic relationships of its phycoflora. From the sampling campaign at Gonzalo Island, 19 taxa of macroalgae were identified. In terms of richness by division, Chlorophyta was represented by 3 species (16\%), Ochrophyta by 7 taxa (37\%), and Rhodophyta by 9 taxa, representing the $47 \%$ of the identified flora. Thus, the present study combines our new registers together with the previous findings, providing an updated cadaster of macroalgae of the archipelago, suggesting a total of 86 taxa. Overall, it can be stated that all registered species of the archipelago show a subAntarctic affinity.

\section{Key words:}

macroalgae, subantarctic,

Diego Ramírez, geographic affinity.

\section{INTRODUCCIÓN}

Las ecorregiones marinas de canales y fiordos del sur de Chile (sensu Spalding et al. 2007) y terrestre subantártica de Magallanes (sensu Rozzi et al. 2012) representan el límite extremo de latitud sur para la distribución de numerosos grupos de organismos a nivel de especies, géneros, familias $e$ incluso órdenes y clases (Rozzi et al. 2007). En los ecosistemas costeros sobresalen los grandes "bosques submarinos" de algas pardas, conocidos internacionalmente como kelps, que alcanzan enormes extensiones y biomasa ofreciendo un hábitat crítico (Stachowicz, 2001), proporcionando refugio, lugar de reproducción y/o alimento para muchas especies de invertebrados y vertebrados, incluyendo especies de interés comercial como erizo, centolla, ostión, calamar y peces (Ríos et al. 2007; Mansilla et al. 2009; Mansilla \& Ávila, 2011; Rosenfeld et al. 2014; Marambio et al. 2016). Los grandes bosques de algas pardas pertenecen a los órdenes Laminariales y Fucales, representados en las costas de altas latitudes en ambos hemisferios. En el caso del Hemisferio Sur, el punto de distribución más austral de representantes de las formaciones de kelp se encuentra en el archipiélago Diego Ramírez, donde se registran las especies Lessonia flavicans, Lessonia searlesiana y Macrocystis pyrifera, además de la especie Durvillaea antarctica, un alga parda de gran tamaño.

El archipiélago Diego Ramírez se encuentra ubicado en el Paso Drake a $112 \mathrm{~km}$ al SO del Cabo de Hornos y a $80 \mathrm{~km}$ al SW de los islotes Ildefonso (Schlatter \& Riveros, 1997); actualmente forma parte del Parque Marino Islas Diego Ramírez-Paso Drake y geográficamente se encuentra en el extremo más austral de Sudamérica. Este archipiélago está formado por dos grupos de islotes, rocas y arrecifes, separados entre sí por una extensión de $3,7 \mathrm{~km}$. El grupo principal incluye a las islas Bartolomé y Gonzalo, separadas por el canal Nodales (Pisano, 1972). Las costas de ambas islas son escarpadas, con una gran pendiente y presentan un alto grado de erosión debido a las constantes marejadas del lugar como consecuencia de la gran influencia oceánica (Schlatter \& Riveros, 1997).

Desde un punto de vista oceanográfico, el extremo sur de las costas del Parque Nacional Cabo de Hornos y las aguas al sur de éste presentan una columna de agua con una estructura casi homogénea debido a los fuertes vientos y mareas típicas de esta zona (Valdenegro \& Silva, 2003).

Desde un punto de vista biogeográfico, el grupo de islas del archipiélago Diego Ramírez es el punto más austral de la provincia Magallánica (Spalding et al. 2007) y se encuentra al borde de la plataforma continental en el ecotono con el Paso Drake surcado por la Corriente Circumpolar Antártica (Rozzi et al. 2017). Con respecto a la biota que se encuentra registrada para esta zona, específicamente macroalgas, para el 
archipiélago Diego Ramírez se ha reportado un total de 79 taxa de macroalgas (Contreras et al. 1983; Mansilla \& Navarro, 2003). En su análisis, Contreras y colaboradores (1983) concluyen que el archipiélago Diego Ramírez presenta una mayor afinidad ficológica con el Continente Antártico que con la Provincia Magallánica y otras provincias de Sudamérica. Estos autores documentan la presencia de la especie de alga parda Desmarestia menziesii, que presenta una distribución antártica y nunca antes había sido registrada en la provincia de Magallanes (Ramírez, 2010). Pese al interés biogeográfico de las macroalgas del archipiélago Diego Ramírez, desde los trabajos de Contreras et al. (1983) y Mansilla y Navarro, (2003), no ha habido nuevos catastros que permitan evaluar si este archipiélago podría representar el extremo norte de distribución de especies antárticas y/o el extremo sur de especies magallánicas.

Por otra parte, las comunidades de macroalgas, en particular las especies de kelp presentes en las islas Diego Ramírez, son las más australes del mundo, siendo además las más próximas al continente antártico. Estos kelps presentan un importante rol como ingeniero ecosistémico, proporcionando una gama de importantes funciones y servicios ecológicos y socioeconómicos (Rosenfeld et al. 2014).

Dentro de estos servicios, se destaca el 'carbono azul' que es captado por las poblaciones de macroalgas, almacenado como biomasa fresca y posteriormente secuestrado en los sedimentos de la vegetación costera; este ciclo actualmente está siendo considerado un servicio de regulación del clima de importancia mundial (Reid \& Mooney, 2005). Sin embargo, la importancia de las macroalgas, como los bosques de algas pardas o kelps, en las evaluaciones de carbono azul, ha sido relativamente poco considerada (Duarte, 2016). Es evidente que la función de las macroalgas en el almacenamiento de carbono podría ser de gran relevancia (Krause-Jensen \& Duarte, 2016). Es por eso que conocer el estado actual de la comunidad de macroalgas en el recién creado Parque Marino Islas Diego Ramírez-Paso Drake es de suma relevancia, puesto que estas islas podrian no solamente servir como un sumidero de carbono, sino también un reservorio genético de especies de interés comercial.
El objetivo de este trabajo es actualizar el catastro de macroalgas existentes en el archipiélago Diego Ramírez con el fin de detectar nuevos taxa que pudieran estar presentes en estas islas, y así contribuir con un listado más actualizado de la comunidad de macroalgas, además de un mejor análisis de las relaciones biogeográficas de su ficoflora.

\section{MATERIALES Y MÉTODOS}

Este trabajo abarcó las costas rocosas de la isla Gonzalo, localizada en el margen austral del Parque Marino Islas Diego Ramírez-Paso Drake, en la latitud $56^{\circ} 31^{\prime} 16,8^{\prime \prime} S$ y longitud $68^{\circ} 42^{\prime} 53,5^{\prime \prime O}$ (Fig. 1). Las costas rocosas ubicadas en este archipiélago se caracterizan por su gran exposición al oleaje (Mansilla \& Navarro, 2003), carácter que se asocia con la gran abundancia y cobertura de poblaciones de Durvillaea antarctica (Ramírez \& Santelices, 1991; Cursach et al. 2014; Méndez et al. 2017). Sin embargo, también es posible encontrar algunas bahías protegidas localizadas en las costas nororientales de la isla Gonzalo, las que han sido menos exploradas desde el punto de vista ficológico (Schlatter \& Riveros, 1997).

La isla Gonzalo se caracteriza por presentar un sustrato rocoso con gran pendiente y alto grado de erosión debido a las constantes marejadas del lugar, además, predominan los sustratos de terrazas formadas por grandes extensiones rocosas que pueden presentar una alta pendiente, generando sitios estables que constituyen un hábitat apropiado para macroalgas y fauna bentónica (Rosenfeld \& Ojeda, 2015). También se encuentran ambientes inestables como playas de grava, por ejemplo, en la zona de desembarco de la isla Gonzalo donde la inestabilidad ocasiona que sea muy difícil para los organismos bentónicos fijarse en la superficie de este tipo de sustrato. No obstante, a la fecha no se han reportado playas de fondos blandos en las costas de esta isla. En general, los ambientes costeros de este archipiélago, al igual que lo descrito para la isla Hornos, están controlados principalmente por los disturbios físicos naturales, tales como el alto hidrodinamismo producto de la exposición al oleaje (Guzmán \& Ríos, 1981; Schlatter \& Riveros, 1997).

La colecta de macroalgas se realizó durante la estación de primavera del año 2016. Durante 

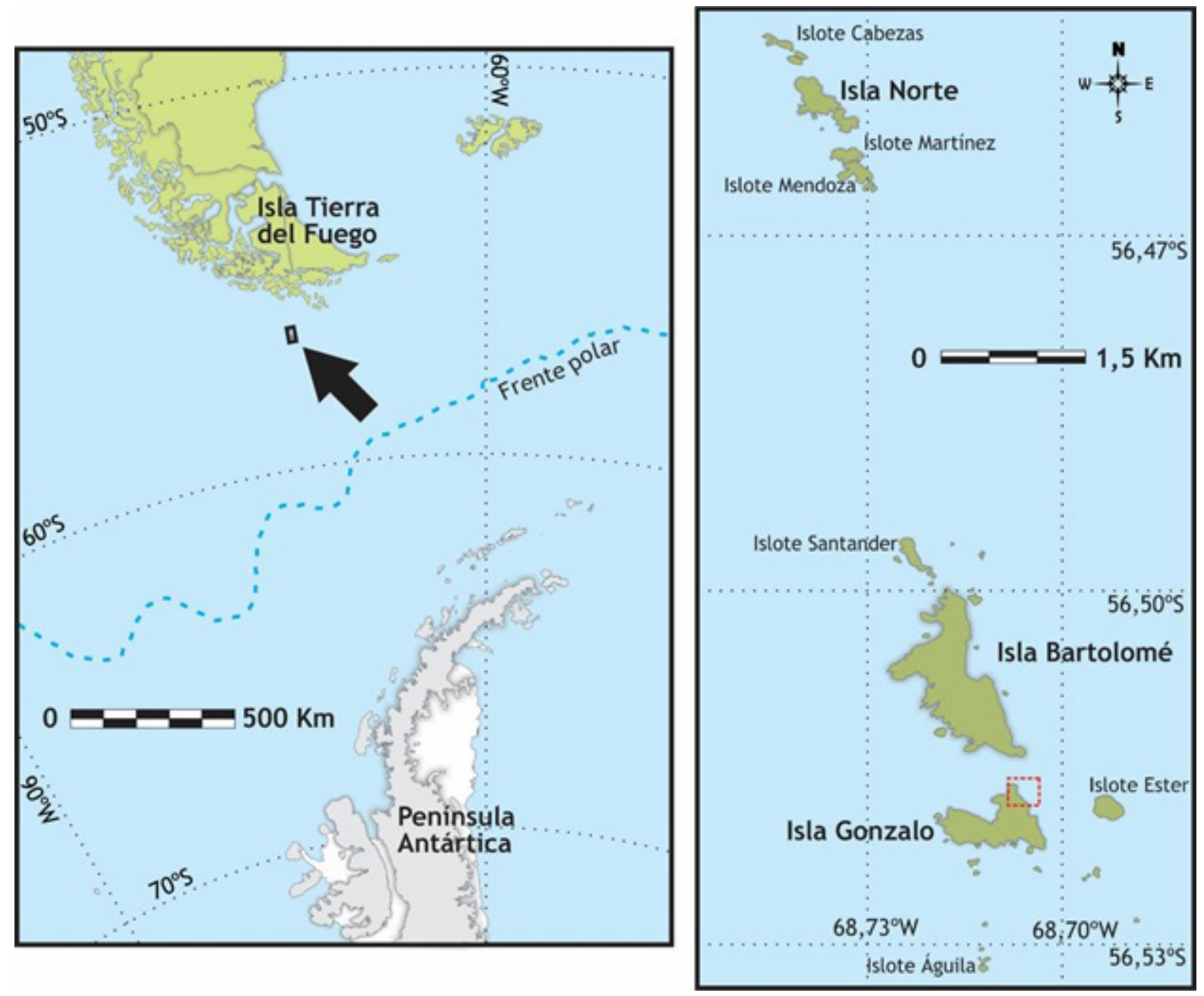

Fig. 1. Mapa sitio de estudio Isla Gonzalo latitud $56^{\circ} 31^{\prime} 16,8^{\prime \prime}$ y y longitud $68^{\circ} 42^{\prime} 53,5^{\prime \prime} \mathrm{O}$, ubicado dentro del Parque Marino Islas Diego Ramírez-Paso Drake.

marea baja se efectuó una exhaustiva inspección visual mediante un transecto perpendicular a la línea de costa desde la zona del intermareal superior hasta $1 \mathrm{~m}$ de profundidad, se colectó material in situ mediante extracción manual y espátula. Las macroalgas fueron conservadas en bolsas plásticas rotuladas y congeladas en un freezer a $-20^{\circ} \mathrm{C}$ en el Laboratorio de Ecosistemas Antárticos y Subantárticos (LEMAS) de la Universidad de Magallanes para su posterior clasificación e identificación, las excicatas elaboradas fueron depositadas en el Herbario de LEMAS, con el fin de realizar un listado actualizado de los taxa presentes en el archipiélago Diego Ramírez. El material colectado en terreno fue comparado con los informes de Contreras et al. (1983) y Mansilla y Navarro (2003).

\section{RESULTADOS Y DISCUSIÓN}

De la campaña realizada en la isla Gonzalo se identificó un total de 19 taxa de macroalgas. En términos de riqueza por división, Chlorophyta estuvo representada por 3 especies (16\%), Ochrophyta por 7 taxa (37\%) y Rhodophyta por 9 taxa, representando el $47 \%$ de la flora identificada (Fig. 2A). Estos 19 taxa incluyeron 14 familias (Tabla 1), siendo la familia Gigartinaceae la más representativa con un registro de 4 especies $(\mathrm{S}=4)$, seguida por la familia Desmarestiaceae $(S=3)$. 
Tabla 1. Lista sistemática de los taxa de macroalgas reportados para el archipiélago Diego Ramírez.

(1) de Contreras et al. (1983), (2) Mansilla y Navarro (2003) y (*) este estudio.

\begin{tabular}{|c|c|c|}
\hline \multicolumn{3}{|l|}{ CHLOROPHYTA } \\
\hline \multicolumn{3}{|l|}{ Orden Bryopsidales } \\
\hline \multirow[t]{2}{*}{ Familia Bryopsidaceae } & Bryopsis plumosa (Hudson) C. Agardh & 2 \\
\hline & Bryopsis rosea Gaudichaud ex C. Agardh & 1 \\
\hline \multirow[t]{3}{*}{ Familia Codiaceae } & Codium dimorphum Svedelius & 1 \\
\hline & Codium fragile (Suringar) Hariot & $*$ \\
\hline & Codium subantarcticum P. C. Silva & 2 \\
\hline \multicolumn{3}{|l|}{ Orden Cladophorales } \\
\hline \multirow[t]{4}{*}{ Familia Cladophoraceae } & Cladophora sp. & 1,2 \\
\hline & Rhizoclonium ambiguum (J. D. Hooker \& Harvey) Kützing & $*$ \\
\hline & Rhizoclonium riparium (Roth) Harvey & 1 \\
\hline & Rhizoclonium tortuosum (Dillwyn) Kützing & 2 \\
\hline \multicolumn{3}{|l|}{ Orden Ulotrichales } \\
\hline \multirow[t]{3}{*}{ Familia Ulotrichaceae } & Acrosiphonia arcta (Dillwyn) Gain & $*$ \\
\hline & Spongomorpha sp. & 1 \\
\hline & Ulothrix flacca (Dillwyn) Thuret & 2 \\
\hline \multicolumn{3}{|l|}{ Orden Ulvales } \\
\hline Familia Kornmanniaceae & Blidingia minima (Nägeli ex Kützing) Kylin & 2 \\
\hline \multirow[t]{3}{*}{ Familia Ulvaceae } & Ulva bulbosa Pasilot de Beauvois & 1,2 \\
\hline & Ulva intestinalis Linnaeus & 1,2 \\
\hline & Ulva lactuca Linnaeus & 1,2 \\
\hline \multicolumn{3}{|l|}{ OCHROPHYTA } \\
\hline \multicolumn{3}{|l|}{ Orden Desmarestiales } \\
\hline \multirow[t]{5}{*}{ Familia Desmarestiaceae } & Desmarestia rossii J.D.Hooker \& Harvey & 1 \\
\hline & Desmarestia ligulata (Stackhouse) J. V. Lamoroux & $1, *$ \\
\hline & Desmarestia confervoides (Bory) M. E. Ramírez \& A. F. Peters & $1,2, "$ \\
\hline & Desmarestia chordalis J. D. Hooker \& Harvey & $*$ \\
\hline & Desmarestia menziesii J. Agardh & 1 \\
\hline \multicolumn{3}{|l|}{ Orden Ectocarpales } \\
\hline \multirow[t]{2}{*}{ Familia Acinetosporaceae } & Geminocarpus geminatus (J.D.Hooker \& Harvey) Skottsberg & 1 \\
\hline & Pylaiella littoralis (Linnaeus) Kjellman & 1 \\
\hline Familia Adenocystaceae & Adenocystis utricularis (Bory) Skottsberg & 1,2 \\
\hline
\end{tabular}


Continuación Tabla 1

\begin{tabular}{|c|c|c|}
\hline \multicolumn{3}{|l|}{ CHLOROPHYTA } \\
\hline \multicolumn{3}{|l|}{ Orden Bryopsidales } \\
\hline Familia Chordariaceae & Chordaria magellanica Kylin & 2 \\
\hline \multirow[t]{2}{*}{ Familia Ectocarpaceae } & Ectocarpus siliculosus (Dillwyn) Lyngbye & 1 \\
\hline & Ectocarpus sp. & 1 \\
\hline Familia Scythosiphonaceae & Scytosiphon lomentaria (Lyngbye) Link & 1, , \\
\hline \multicolumn{3}{|l|}{ Orden Fucales } \\
\hline Familia Durvillaeaceae & Durvillaea antarctica (Chamisso) Hariot & $1,2,{ }^{*}$ \\
\hline \multicolumn{3}{|l|}{ Orden Laminariales } \\
\hline \multirow[t]{2}{*}{ Familia Lessoniaceae } & Lessonia flavicans Bory & $1,2, *$ \\
\hline & Lessonia searlesiana Asensi \& de Reviers & 1,2 \\
\hline Familia Laminariaceae & e Macrocystis pyrifera (Linnaeus) C. Agardh & $1,2, *$ \\
\hline
\end{tabular}

Orden Ahnfeltiales

Familia Ahnfeltiaceae Ahnfeltia plicata (Hudson) Fries

Orden Balliales

Familia Balliaceae Ballia callitricha (C.Agardh) Kützing

Orden Bangiales

\begin{tabular}{llc} 
Familia Bangiaceae & Pyropia columbina (Montagne) W.A.Nelson & 2 \\
\cline { 2 - 3 } & Pyropia endiviifolia (A.Gepp \& E.Gepp) H.G.Choi \& M.S.Hwang & 1 \\
\cline { 2 - 3 } Pyropia sp. & $2, *$
\end{tabular}

Orden Bonnemaisoniales

Familia Bonnemaisoniaceae Ptilonia magellanica (Montagne) J. Agardh

Orden Ceramiales

Familia Ceramiaceae

Callithamnion montagnei J.D.Hooker

Callithamnion sp. 1

$\begin{array}{lr}\text { Ceramium dozei Hariot } & 1,2\end{array}$

Ceramium virgatum Roth $\quad 1,2$

Ceramium sp.

Pterothamnion antarcticum (Kylin) R.L.Moe \& P.C.Silva 1

Pterothamnion simile (J.D.Hooker \& Harvey) Nägeli 1 
Continuación Tabla 1

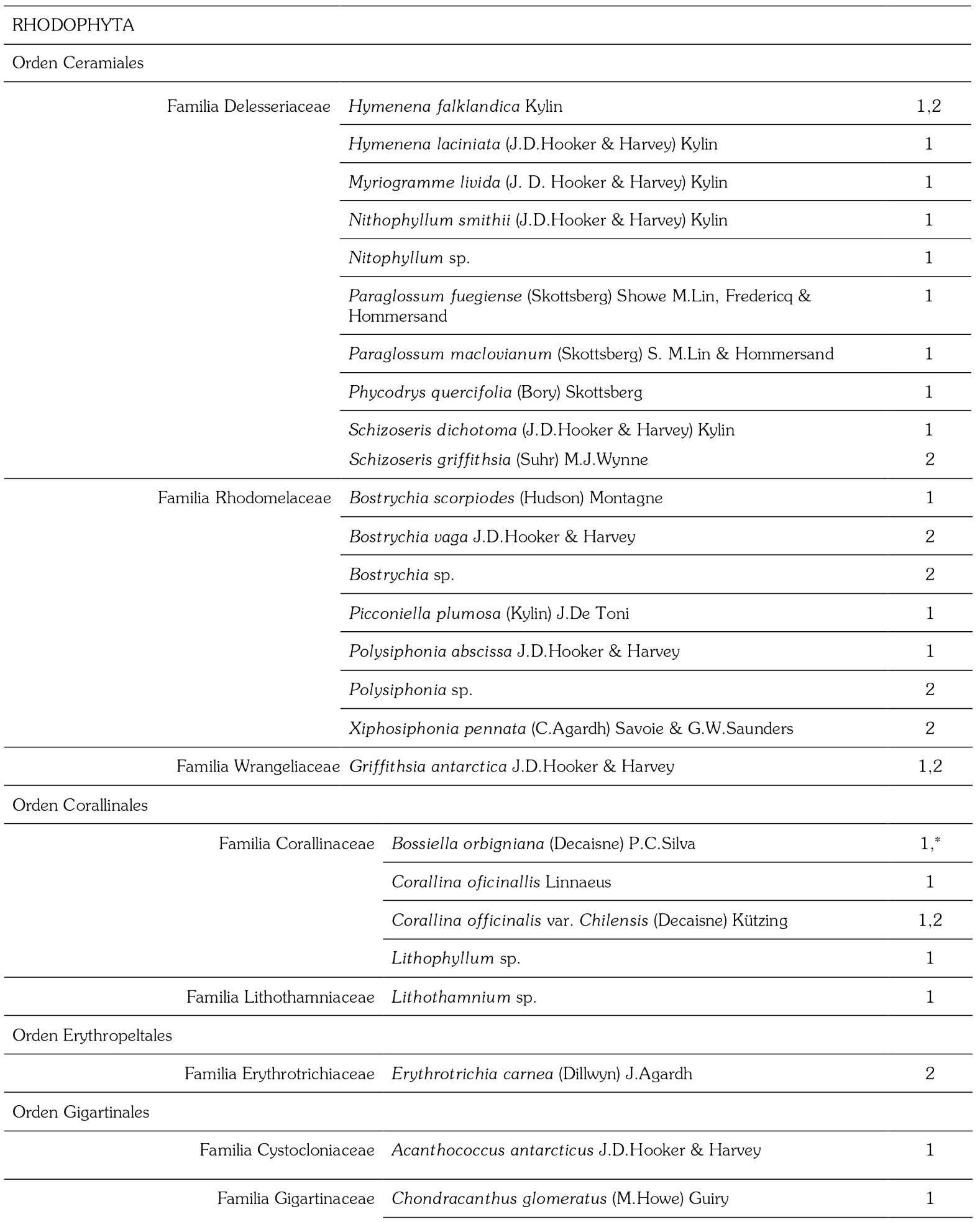


Continuación Tabla 1

\begin{tabular}{|c|c|c|c|}
\hline RHODOPHYTA & & & \\
\hline Orden Gigartinale & & & \\
\hline & & Gigartina skottsbergii Setchell \& N.L.Gardner & $1,2, *$ \\
\hline & & Iridaea cordata (Turner) Bory de Saint-Vincent & $1,2, *$ \\
\hline & & Mazzaella laminarioides (Bory) Fredericq & $2, *$ \\
\hline & & Sarcothalia crispata (Bory) Leister & $1, *$ \\
\hline & Familia Kallymeniaceae & Callophyllis variegata (Bory) Kützing & 1,2 \\
\hline Orden Halymenia & & & \\
\hline & Familia Halymeniaceae & Grateloupia doryphora (Montagne) M.Howe & 2 \\
\hline & & Pachymenia lusoria (Greville) J.Agardh & $*$ \\
\hline & & Prionitis lanceolata (Harvey) Harvey & 1 \\
\hline
\end{tabular}

Orden Hapalidiales

Familia Mesophyllaceae Clathromorphum sp.

\begin{tabular}{|c|c|c|c|}
\hline \multicolumn{4}{|l|}{ Orden Hildenbrandiales } \\
\hline & Familia Hildenbrandiaceae & Hildenbrandia lecannellieri Hariot & 1 \\
\hline \multicolumn{4}{|l|}{ Orden Nemaliales } \\
\hline & Familia Scinaiaceae & Nothogenia fastigiata (Bory) P.G.Parkinson & 1,2 \\
\hline \multicolumn{4}{|l|}{ Orden Plocamiales } \\
\hline & Familia Plocamiaceae & Plocamium cartilagineum (Linnaeus) P.S.Dixon & 1,2 \\
\hline & Familia Sarcodiaceae & Trematocarpus antarcticus (Hariot) Fredericq \& R.L. Moe & $*$ \\
\hline \multicolumn{4}{|l|}{ Orden Rhodymeniales } \\
\hline & Familia Rhodymeniaceae & Rhodymenia coccocarpa (Montagne) M.J.Wynne & 2 \\
\hline
\end{tabular}

Actualmente el archipiélago Diego Ramírez forma parte del Parque Marino Islas Diego RamírezPaso Drake, representando el archipiélago más austral del continente sudamericano y ha sido comparativamente poco explorado. Los dos estudios previos realizados por Contreras et al. (1983) y Mansilla y Navarro (2003) compilaron un total de 79 especies para este archipiélago.

Nuestro estudio colectó un total de 19 especies, de las cuales siete taxa no habían sido reportados para este archipiélago en los trabajos mencionados anteriormente. Los siete nuevos registros para este archipiélago son tres especies de Chlorophyta,
Codium fragile (Suringar) Hariot, Rhizoclonium ambiguum (J. D. Hooker \& Harvey) Kützing y Acrosiphonia arcta (Dillwyn) Gain; una especie de Ochrophyta, Desmarestia chordalis (Fig. 3A, B) J. D. Hooker \& Harvey; y tres especies de Rhodophyta, Pachymenia lusoria (Greville) J. Agardh, Ceramium sp., y Trematocarpus antarcticus (Hariot) Fredericq \& R. L. Moe (Fig. 4A, B).

Con respecto a los 19 taxa registrados en este estudio, los trabajos de Ramírez (2010) y Boraso de Zaixo (2004) estarían indicando que aquéllos presentan una mayor afinidad con la 


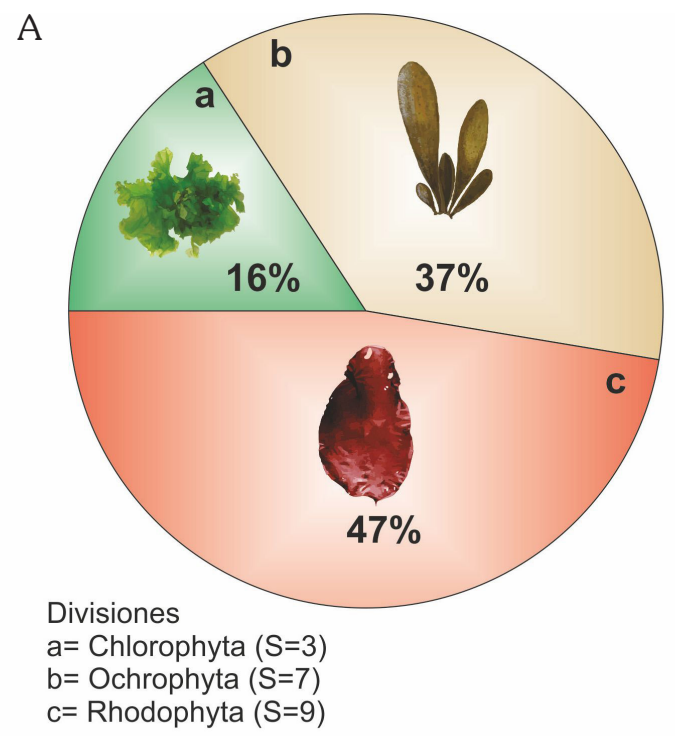

B

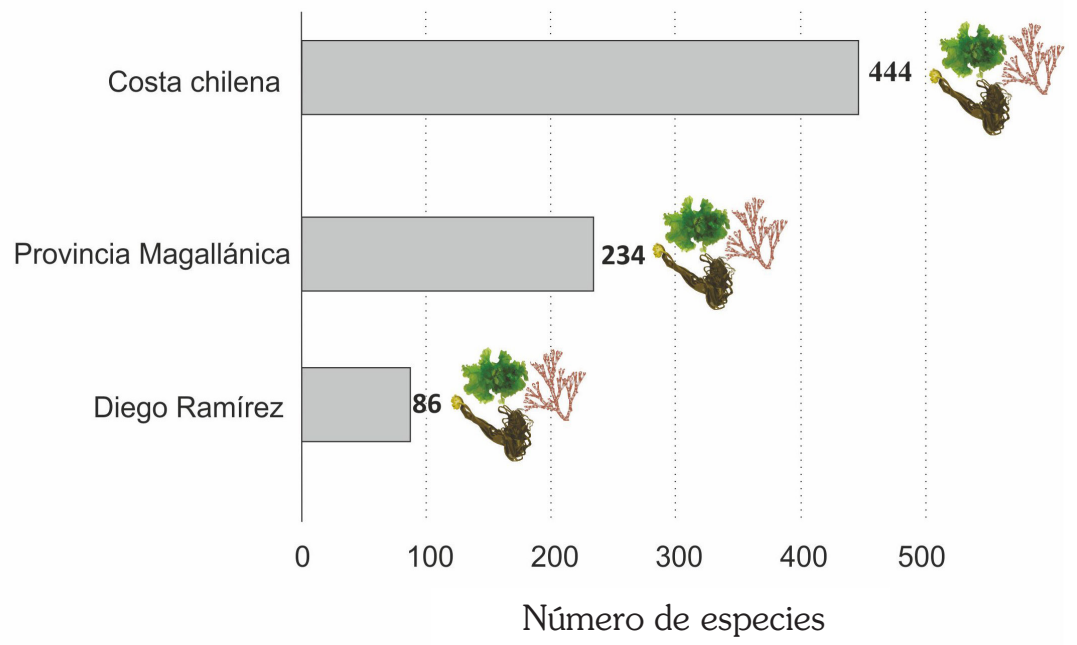

Fig. 2. A) Porcentaje de especies reportadas en este estudio. B) Número de especies de macroalgas reportadas para la costa chilena, provincia Magallánica e islas Diego Ramírez.

región subantártica. No obstante, se destaca que las especies $A$. arcta, P. lusoria y T. antarcticus también presentan distribución antártica (Papenfuss, 1964; Guiry \& Guiry, 2018). Esta afinidad con la región subantártica concuerda con el trabajo de Ramírez (2010) que menciona que las localidades de Magallanes - Tierra del Fuego y Malvinas-Falkland presentan una similitud de un $60 \%$ en su flora ficológica, aislando a la región antártica con una similitud de un $23 \%$.

Con estos nuevos registros, nuestro estudio genera un catastro actualizado de macroalgas para el archipiélago que incluye un total de 86 taxa de macroalgas (Tablas 1). Estos taxa pertenecen en un $18 \%$ a la división Chlorophyta (16 taxa), un $19 \%$ a la división Ochrophyta (16 taxa) y un $63 \%$ a la división Rhodophyta (54 taxa).

Para Chile se han registrado 444 taxa de macroalgas, de los cuales 234 están presentes en la zona de Magallanes y Tierra del Fuego, mientras que la flora marina bentónica de la región de Magallanes presenta el $50 \%$ de las macroalgas descritas para el territorio chileno (Ramírez, 2010). Con nuestro estudio, el número de taxa registrados 


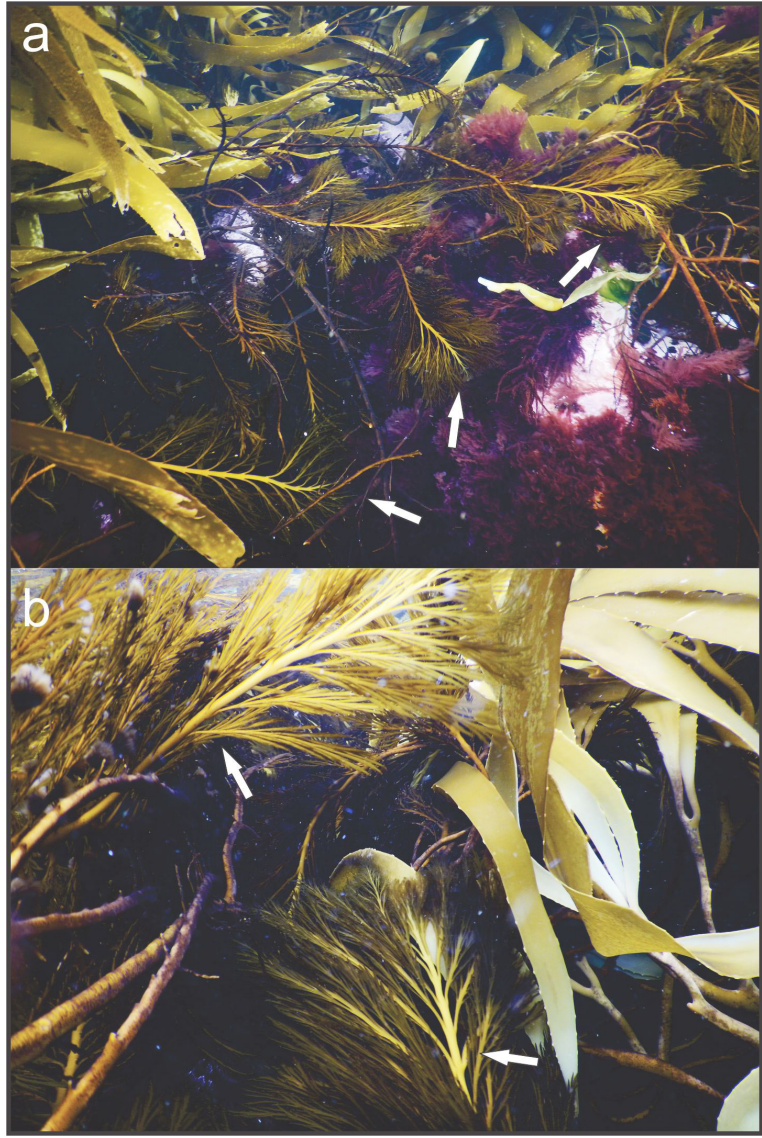

Fig. 3. Fotografía en terreno del alga parda Desmarestia chordalis registrada en el submareal somero de la isla Gonzalo, archipiélago Diego Ramírez. A) Las flechas indican la zona apical de las frondas del alga. B) Las flechas indican el talo erecto y cilíndrico de ejemplares adultos.

para el archipiélago Diego Ramírez aumentó de 79 a 86 taxa, cifra que representa el $37,6 \%$ de macroalgas descritas para la región de Magallanes (Fig. 2b). Más aún, el 20\% de los taxa de macroalgas registrados para Chile se encuentra representado en este pequeño archipiélago del extremo austral del continente americano.

En la ecorregión de canales y fiordos del sur gran parte del conocimiento sobre macroalgas se originó a partir de expediciones científicas del siglo XVIII (Ramírez, 2010). En contraste, trabajos actuales enfocados en la caracterización y diversidad de ensambles costeros de macroalgas son escasos en comparación a otros grupos taxonómicos. Además, la mayor parte de los estudios ficológicos se ha centrado en localidades ubicadas en el estrecho de Magallanes, por lo cual es importante

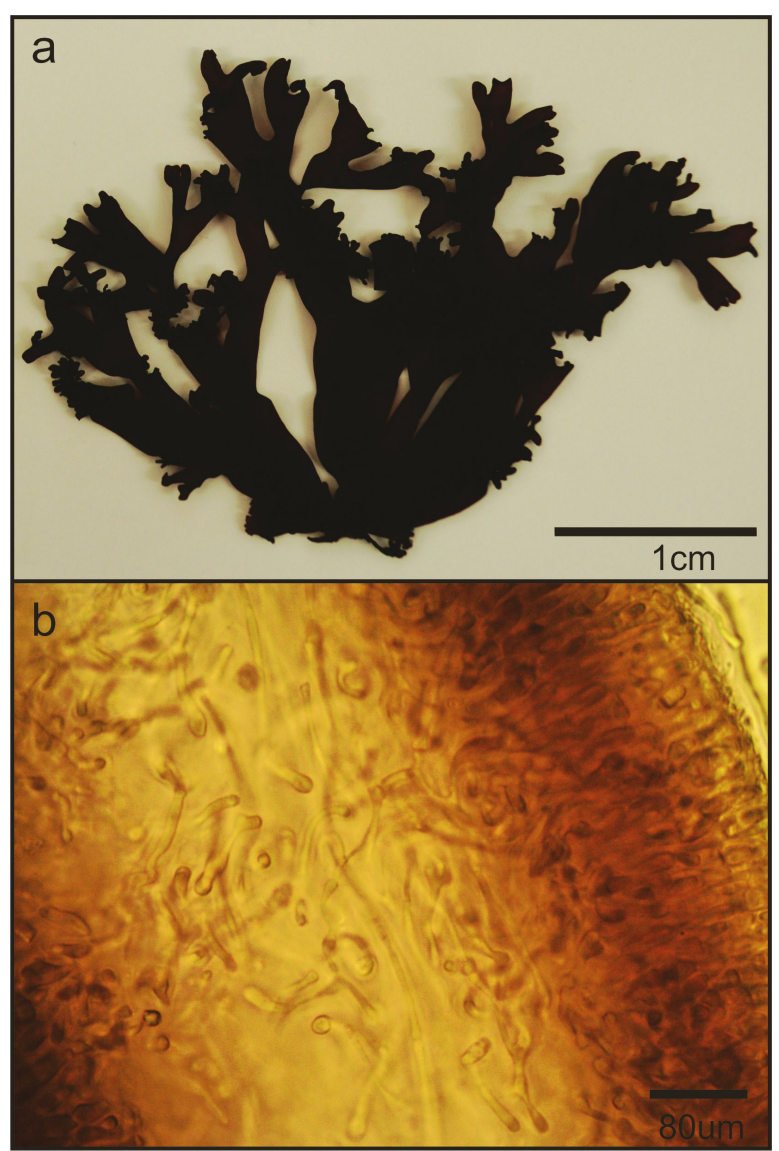

Fig. 4. Fotografía del alga roja Trematocarpus antarcticus registrada en el submareal somero de la isla Gonzalo, archipiélago Diego Ramírez. A) Vista general del talo aplanado, con presencia de ramificaciones dicotómicas. B) Corte transversal donde se aprecia la médula de consistencia filamentosa.

destacar la contribución de nuestro estudio puesto que identifica a la isla Gonzalo como la localidad con la mayor riqueza de macroalgas informadas hasta ahora en costas magallánicas (Tabla 1).

Con respecto a la conservación de la biodiversidad, es relevante mencionar que, a la fecha, en la isla Gonzalo no hay registro de especies de macroalgas invasoras, por lo tanto, esta zona es una de las pocas costas libres de especies exóticas. No obstante, para el resto de la Provincia Magallánica se ha reportado la presencia del alga exótica Polysiphonia morrowii (Kim et al. 2004; Mansilla et al. 2013). Esta macroalga exótica ha llegado a ser un componente común en ensambles intermareales del estrecho de Magallanes y otros sitios de la Provincia Magallánica (Rosenfeld et al. 2018). 
Respecto a la afinidad florística de las macroalgas del archipiélago Diego Ramírez con la ficoflora de las costas antárticas, Contreras et al. (1983) destacan la presencia de la especie Desmarestia menziesii, la cual se encuentra distribuida en el continente antártico e islas de la península (Guiry \& Guiry, 2018). Sin embargo, Mansilla y Navarro (2003) no registraron esta especie y en nuestro estudio tampoco fue localizada a pesar de los esfuerzos dirigidos en su búsqueda. Según una serie de trabajos (Papenfuss, 1964; Ramírez \& Santelices, 1991; Mendoza \& Nizovoy, 2000; Boraso et al. 2003; Boraso, 2004; Ramírez, 2010), el actual listado de algas referidas para el archipiélago Diego Ramírez presenta una mayor afinidad subantártica, lo cual se ve reflejado en los componentes florísticos magallánicos. Es importante destacar la abundancia de los bosques de kelp formados por grandes macroalgas del Orden Laminariales (Macrocystis pyrifera, Lessonia searlesiana y L. flavicans) y macroalgas de gran tamaño del Orden Fucales (Durvillaea antarctica), estas especies alcanzan su extremo sur de distribución en los ecosistemas costeros del Parque Marino Islas Diego Ramírez-Paso Drake. Estos bosques de macroalgas pardas proveen además un hábitat clave para la alimentación, reproducción y refugio de numerosos taxa de origen sudamericano. Por lo tanto, nuestro estudio aporta antecedentes que refuerzan el nexo de la ficoflora y la biodiversidad del archipiélago Diego Ramírez con la Provincia Magallánica.

Dado los resultados obtenidos, se puede concluir que este trabajo es el tercer registro de la flora marina bentónica para la isla Gonzalo. Este estudio ha sido realizado después de 15 años del último muestreo realizado en la zona por Mansilla y Navarro (2003), lo cual claramente indica el escaso esfuerzo de muestreo que ha presentado esta área urante los últimos años, evidenciando aún más la presencia de notables vacíos de información de la flora marina en el Parque Marino Islas Diego Ramírez-Paso Drake. Con los datos obtenidos en este estudio y los recopilados de trabajos anteriores, se destaca la presencia de flora marina con afinidad subantártica. Sin embargo, cabe destacar que algunas especies tienen presencia tanto en la región subantártica, islas antárticas y península Antártica; no obstante, no se pudo registrar durante este estudio flora marina descrita exclusivamente para Antártica, como es el caso de Desmarestia menziesii, especie registrada en estos confines por Contreras et al. (1983). En concordancia con lo anterior, el archipiélago Diego Ramírez destaca como el último punto de distribución en latitudes altas de una serie de especies que habitan la región de Magallanes o que presentan distribución subantártica.

\section{AGRADECIMIENTOS}

Los autores agradecen al Consejo Nacional de Investigación en Ciencia y Tecnología (CONICYT) del programa FONDECYT otorgado 1110875 a Andrés Mansilla, al Proyecto Conicyt PIA Apoyo CCTE AFB170008 a través del Instituto de Ecología y Biodiversidad (IEB) y al programa FONDECYT 1161358; A. M. agradece el apoyo de Proyecto FONDECYT 1180433. Un especial agradecimiento a la Armada de Chile, al Comandante en Jefe de la III Zona Naval, Contraalmirante Ivo Brito, al Jefe de Estado Mayor General, Vicealmirante José Miguel Rivera, al Comité Oceanográfico Nacional y a la Comandancia del Distrito Naval Beagle, como también a la tripulación de la nave OPV 83 Marinero Fuentealba, al personal de traslado en helicóptero y de logística institucional y al personal del Faro de la isla Gonzalo por su invaluable apoyo para el desarrollo de investigación de terreno en el sitio de estudios ecológicos permanente, implementado en el archipiélago Diego Ramírez.

\section{BIBLIOGRAFÍA}

Boraso, A. (2004). Chlorophyta marinas de la Argentina. Historia Natural, 3(2), 95-119. Boraso, A., Rico, A., Perales, S., Pérez, L., \& Zalazar, H. (2003). Una guía ilustrada algas marinas de la Patagonia. Buenos Aires, Argentina: Fundación de Historia Natural, Edición Vázquez Mazzini.

Contreras, D., Schlatter, R., \& Ramírez, C. (1983). Flora ficológica de las Islas Diego Ramírez (Chile). Serie Científica INACH, 30, 1326.

Cursach, J., Suazo, C., Rau, J., Niklitschek, E., \& Vilugrón, J. (2014). Observaciones sobre el 
pingüino de penacho amarillo Eudyptes $c$. chrysocome en isla Gonzalo, archipiélago Diego Ramírez, Chile. Revista de Biología Marina y Oceanografía, 49(2), 467-472.

Duarte, C. M. (2016). Reviews and syntheses: Hidden Forests, the role of vegetated coastal habitats on the ocean carbon budget. Biogeosciences Discussions, 1981, 1-17.

Guiry, M. D., \& Guiry, G. M. (2018). AlgaeBase. World-wide electronic publication. Galway: National University of Ireland. http://www. algaebase.org; searched on 09 May 2018.

Guzmán, L., \& Ríos, C. (1981). Estructura del conjunto de macroorganismos de una playa de bloques y cantos rodados de Isla Wollaston, Archipiélago del Cabo de Hornos. Anales del Instituto de la Patagonia, 12, 257-271.

Kim, M., Yang, E. C., \& Mansilla, A. (2004). Recent introduction of Polysiphonia morrowii (Ceramiales, Rhodophyta) to Punta Arenas, Chile. Botánica Marina, 47, 389-394.

Krause-Jensen, D., \& Duarte, C.M. (2016). Substantial role of macroalgae in marine carbon sequestration. Nature Geoscience, 9, 737-742.

Mansilla, A., \& Navarro, N. (2003). Contribución al estudio de la flora ficológica de las islas Diego Ramírez (Chile). En A. Mansilla, C. Werlinger, \& N. Navarro (Eds.), Memorias curso internacional de postgrado $y$ especialización de macroalgas en ambientes subantárticos (pp. 85-89). Punta Arenas: Ediciones Universidad de Magallanes.

Mansilla, A., \& Ávila, M. (2011). Using Macrocystis pyrifera (L.) C. Agardh from southern Chile as a source of applied biological compounds. Brasilian Farmacognosia, 21, 262-267.

Mansilla, A., Ávila, M., Cáceres, J., Palacios, M., Navarro, N., Cañete, I., \& Oyarzún, S. (2009). Diagnóstico bases biológicas explotación sustentable Macrocystis pyrifera, (Huiro). XII Región Código BIP 30060262-0. Gobierno Regional de Magallanes y Antártica Chilena. Informe de proyecto. Punta Arenas: Universidad de Magallanes.

Mansilla, A., Ávila, M., Ramírez, M. E., Rodríguez, J. P., Rosenfeld, S., Ojeda, J., \& Marambio,
J. (2013). Macroalgas marinas bentónicas del submareal somero de la ecorregión subantártica de Magallanes, Chile. Anales del Instituto de la Patagonia, 41(2), 49-62.

Marambio, J., Rosenfeld, S., Ojeda, J., \& Mansilla, A. (2016). Variación estacional en la composición de ensambles sublitorales de macroalgas asociadas al alga roja Gigartina skottsbergii Setchell \& Gardner, en el Estrecho de Magallanes, Chile. Anales del Instituto de la Patagonia, 44(2), 5-22.

Méndez, F., Tala, F., Rautenberger, R., Ojeda, J., Rosenfeld, S., Rodríguez, J. P., Marambio, J., Ocaranza, P., \& Mansilla, A. (2017). Morphological and physiological differences between two morphotypes of Durvillaea antarctica (Phaeophyceae) from the subAntarctic ecoregion of Magallanes, Chile. Journal of Applied Phycology, 29, 25572565.

Mendoza, M. L., \& Nizovoy, A. (2000). Géneros de macroalgas marinas de la Argentina, fundamentalmente de Tierra del Fuego. Argentina: Editado por el Poder Legislativo de la Provincia de Tierra del Fuego, Antártida e Islas del Atlántico Sur.

Papenfuss, G. F. (1964). Catalogue and bibliography of Antarctic and Sub-Antarctic benthic marine algae. In M. O. Lee (Ed.), Antarctic Research Series. Volume 1. Bibliography of the Antarctic Seas (pp. 1-76). Washington D.C.: American Geophysical Union.

Pisano, E. (1972). Observaciones fito-ecológicas en las islas Diego Ramírez. Anales del Instituto de la Patagonia, 3(1-2), 161-169.

Ramírez, M. E. (2010). Flora marina bentónica de la región austral de Sudamérica y la Antártica. Anales del Instituto de la Patagonia, 38(1), 57-71.

Ramírez, M. E., \& Santelices, B. (1991). Catálogo de las algas marinas bentónicas de la costa del Pacífico temperado de Sudamérica. Monografías Biológicas 5. Santiago, Chile: Pontificia Universidad Católica de Chile.

Reid, W., \& Mooney, H. (2005). The millennium ecosystem assessment: Testing the limits of interdisciplinary and multi-scale science: Essays in honour of Kanchan Chopra. Ecology, Economy and Society, DOI: 
10.1007/978-981-10-5675-8_4

Ríos, C., Arntz, W., Gerdes, D., Mutschke, E., \& Montiel, A. (2007). Spatial and temporal variability of the benthic assemblages associated to the holdfasts of the kelp Macrocystis pyrifera in the Straits of Magellan, Chile. Polar Biology, 31, 89100.

Rosenfeld, S., \& Ojeda, O. (2015). Habitanteshábitats y hábitos marinos. En T. Contador, S. Rosenfeld, J. Ojeda, \& J. Kennedy (Eds.), Historia natural de los invertebrados del Cabo de Hornos (pp. 40-51). Punta Arenas, Chile: Fundación Omora, Universidad de Magallanes.

Rosenfeld, S., Ojeda, J., Hune, M., Mansilla, A., \& Contador, T. (2014). Egg masses of the Patagonian squid Doryteuthis (Amerigo) gahi attached to giant kelp (Macrocystis pyrifera) in the sub-Antarctic ecoregion. Polar Research, 33, 21636.

Rosenfeld, S., Marambio, J., Ojeda, J., Rodríguez, J. P., González-Wevar, C. A., Gerard, K., Contador, T., Pizarro, G., \& Mansilla, A. (2018). Trophic ecology of two coexisting Sub- Antarctic limpets of the genus Nacella: Spatio-temporal variation in food availability and diet composition of Nacella magallanica and $N$. deaurata in the Sub- Antarctic Ecoregion of Magellan. ZooKeys, 738, 1-25.

Rozzi, R., Massardo, F., Mansilla, A., Anderson, C. B., Berghöfer, A., Mansilla, M., Gallardo, M. R., Plana, J., Berghöfer, U., Arango, X., Russell, S., Araya, P., \& Barros, E. (2007). La Reserva de Biosfera Cabo de Hornos: un desafío para la conservación de la biodiversidad e implementación del desarrollo sustentable en el extremo austral de América. Anales del Instituto de la
Patagonia, 35, 55-70.

Rozzi, R., Armesto, J. J., Gutiérrez, J. R., Massardo, F., Likens, G. E., Anderson, C. B., Poole, A., Moses, K. P., Hargrove E., Mansilla, O., Kennedy, J., Willson, M., Jax, K., Jones, C. G., Callicot, J. B., \& Arroyo, M. (2012). Integrating ecology and environmental ethics: Earth stewardship in the southern end of the Americas. BioScience, 62(3), 226-236.

Rozzi, R., Massardo, F., Mansilla, A., Squeo, F. A., Barros, E., Contador, T., Frangopulos, M., Poulin, E.,Rosenfeld, S.,Goffinet, B., González-Weaver, C., MacKenzie, R., Crego, R. D.,Viddi, F., Naretto, J., Gallardo, M. R., Jiménez, J. E., Marambio, J., Pérez, C., Rodríguez, J. P., Méndez, F., Barroso, O., Rendoll, J., Schuttler, E., \& Martinic, M. (2017). Parque Marino Cabo de Hornos - Diego Ramírez. Punta Arenas, Chile: Ediciones Universidad de Magallanes.

Schlatter, R. P., \& Riveros, G. M. (1997). Historia Natural del Archipiélago Diego Ramírez, Chile. Serie Científica INACH, 47, 87112.

Spalding, M. D., Fox, H. E., Allen, G. R., Davidson, N., Ferdaña, Z. A., \& Robertson, J. (2007). Marine ecoregions of the world: A bioregionalization of coastal and shelf areas. BioScience, 57(7), 573-583.

Stachowicz, J. (2001). Mutualism, facilitation, and structure of ecological communities. BioScience, 51(3), 235-246.

Valdenegro, C., \& Silva, N. (2003). Caracterización oceanográfica física y química de la zona de canales y fiordos australes de Chile entre el estrecho de Magallanes y Cabo de Hornos (CIMAR 3 fiordos). Ciencia y Tecnología del Mar, 26(2), 19-60. 